\title{
Relativistic Static Thin Disks with Radial Stress Support
}

\author{
Guillermo A. González* and Patricio S. Letelier ${ }^{\dagger}$ \\ Departamento de Matemática Aplicada - IMECC \\ Universidade Estadual de Campinas \\ 13081-970, Campinas, S.P., Brazil
}

\begin{abstract}
New solutions for static non-rotating thin disks of finite radius with nonzero radial stress are studied. A method to introduce either radial pressure or radial tension is presented. The method is based on the use of conformal transformations.

PACS numbers: 04.20.Jb, 98.62.Hr, 02.30.Em
\end{abstract}

\section{Introduction}

Axially symmetric solutions of Einstein field equations corresponding to disklike configurations of matter are of great astrophysical interest and have been extensively studied. These solutions can be static or stationary and with or without radial pressure. Solutions for static disks without radial pressure were first studied by Bonnor and Sackfield [1], and Morgan and Morgan [2], and with radial pressure by Morgan and Morgan [3], and, in connection with gravitational collapse, by Chamorro, Gregory and Stewart [4. Static disks with radial tension may also be of interest and, although their relevance in

\footnotetext{
*Permanent address: Escuela de Física, Universidad Industrial de Santander, A.A. 678, Bucaramanga, Colombia, e-mail: guillego@uis.edu.co

†e-mail: letelier@ime.unicamp.br
} 
astrophysics is limited, these solutions can be studied as possible sources of exact solutions for axially symmetric static problems.

Static thin disks without radial stress generate a Weyl spacetime. Then they are described by two different metric functions [5, 6]. The stability of these models can be explained by either assuming the existence of hoop stresses or that the particles on the disk plane move under the action of their own gravitational field in such a way that as many particles move clockwise as counterclockwise. This last interpretation is frequently made since it can be invoked to mimic true rotational effects. A large class of statics thin disks solutions were obtained by Letelier and Oliveira [7] using the inverse scattering method. Solutions for self-similar static disks were analyzed by Lynden-Bell and Pineault [8], and Lemos [9]. The superposition of static disks with black holes were considered by Lemos and Letelier [10, 11, 12], and Klein [13]. Recently Bičák, Lynden-Bell and Katz [14] studied static disks as sources of known vacuum spacetimes and Bičák, Lynden-Bell and Pichon [15] found an infinity number of new static solutions.

Static disks with radial stress are described by three metric functions so presenting some degree of complexity and, despite its clear relevance, has been seldom treated in the literature. The fact that the additional metric function can be considered as the real part of an analytic function will be the main ingredient of our method to introduce radial stress. Static disks with radial stress can also be obtained in other ways. Lemos [9] studied self-similar static disks working in spherical polar coordinates and using self-similarity to reduce the Einstein field equations to an ordinary differential equation. Recently, Pichon and Lynden-Bell [16] obtained static disks by making a cut through a Weyl solution above all singularities or sources. The identification of this solution with its mirror image yields disks with radial stress.

In this work we consider static non-rotating thin disks of finite radius with nonzero radial stress. To obtain a well behaved radial stress we find an analytical function compatible with the boundary conditions imposed on the additional metric function by the requirement that the solution be singularity-free outside the disk. We find a general expression for the radial stress that can be written in terms of a square root in such away that by choosing a sign solutions with radial pressure and solutions with radial tension can be obtained. When we take the positive (negative) sign of the square root, the stress is positive (negative) and so we will have disks solutions with radial pressure (tension). 
The plan of the paper is as follows. In Sec. 2 we present the principal aspects of static thin disks solutions with radial stress, the expression for the energy-momentum tensor of the disk and the general solution that leads to disks with radial pressure or with radial tension. In Sec. 3 we study solutions with radial pressure. In Sec. 4 solutions with radial tension are considered. We conclude, in Sec. 5, by summaring our main results.

\section{Static Thin Disks Solutions}

\subsection{The General Solution}

The metric for a static axially symmetric spacetime can be written in quasicylindrical coordinates $(r, \varphi, z)$ in the form [17, 18]

$$
d s^{2}=-e^{\Phi} d t^{2}+R^{2} e^{-\Phi} d \varphi^{2}+e^{\Lambda-\Phi}\left(d r^{2}+d z^{2}\right),
$$

where $R, \Phi$, and $\Lambda$ are functions of $r$ and $z$ only. In the vacuum, the Einstein equations are equivalent to

$$
\begin{aligned}
& R,_{r r}+R,_{z z}=0, \\
& \left(R \Phi,_{r}\right)_{, r}+\left(R \Phi,{ }_{z}\right)_{, z}=0 \\
& \left(R,{ }_{r}^{2}+R,_{z}^{2}\right) \Lambda,_{r}=\frac{1}{2} R R,_{r}\left(\Phi,,_{r}^{2}-\Phi,,_{z}^{2}\right)+R R,,_{z} \Phi,,_{r} \Phi,_{z}+\left(R,{ }_{r}^{2}+R,{ }_{z}^{2}\right){ }_{r}, \\
& \left(R,_{r}^{2}+R,_{z}^{2}\right) \Lambda,_{z}=\frac{1}{2} R R,_{z}\left(\Phi,_{z}^{2}-\Phi,_{r}^{2}\right)+R R,_{r} \Phi,_{r} \Phi,_{z}+\left(R,_{r}^{2}+R,_{z}^{2}\right)_{,},
\end{aligned}
$$

where ()$_{a}=\partial / \partial x^{a}$.

In order to obtain a solution of (2) - (5) representing a thin disk located at $z=0,0 \leq r \leq 1$, we assume that the metric functions $R, \Phi$, and $\Lambda$ are continuous across the disk, but have discontinuous first derivatives in the direction normal to the disk. Althoug the radius of the disk has been taken as unity, a suitable re-scaling of $r$ can be made. We will comeback to this point later. The reflectional symmetry of (2) - (5) with respect to the plane $z=0$ allows us to assume that $R, \Phi$, and $\Lambda$ are even functions of $z$. Hence, $R, z, \Phi, z$ and $\Lambda,_{z}$ are odd functions of $z$. We shall require that they not vanish on the surfaces $z=0^{ \pm}$. 
We now solve the Laplace equation (2) in the region $r \geq 0, z>0$ with the boundary conditions

$$
\begin{aligned}
R(0, z)=0, & z>0, \\
R,_{z}(r, 0)=0, & r>1, \\
R,_{z}(r, 0) \quad \neq 0, & r \leq 1 .
\end{aligned}
$$

Let $\omega=r+i z$. We can consider $R$ as the real part of an analytical function $W(\omega)=R(r, z)+i Z(r, z)$. Due to the discontinuous behavior of $R,_{z}$ across the disk, the Cauchy-Riemann equations imply that $Z$ is discontinuous across the disk while its normal derivative $Z,_{z}$ is continuous. Then, the invariance of reflection in the plane of the disk allows us to assume $W(r,-z)=\bar{W}(r, z)$. We also require that $W(\omega)$ be locally one-to-one in the outer region of the disk.

A simple solution compatible with these conditions is

$$
W(\omega)=\omega \pm \alpha \sqrt{\omega^{2}-1},
$$

where $\alpha$ is a positive constant, and the \pm means that we can take any of the two possible signs of the square root. When $z \geq 0$ we obtain

$$
\begin{aligned}
& R(r, z)=r \pm \alpha\left[\frac{\left(\left|r^{2}-z^{2}-1\right|^{2}+4 r^{2} z^{2}\right)^{\frac{1}{2}}+\left(r^{2}-z^{2}-1\right)}{2}\right]^{\frac{1}{2}} \\
& Z(r, z)=z \pm \alpha\left[\frac{\left(\left|r^{2}-z^{2}-1\right|^{2}+4 r^{2} z^{2}\right)^{\frac{1}{2}}-\left(r^{2}-z^{2}-1\right)}{2}\right]^{\frac{1}{2}} .
\end{aligned}
$$

For $z \leq 0$ we only need to replace $z \rightarrow-z$ in (10) and (11).

In order to solve the other field equations, we make a conformal transformation

$$
r \rightarrow R(r, z), \quad z \rightarrow Z(r, z),
$$

in such a way that

$$
\Phi \rightarrow \tilde{\Phi}=\Phi, \quad \Lambda \rightarrow \tilde{\Lambda}=\Lambda-\ln \left|W^{\prime}\right|^{2}
$$


where $W^{\prime}=d W / d \omega$. With this transformation the field equations take the usual Weyl form [5, 6]

$$
\begin{gathered}
\tilde{\Phi},_{R R}+\frac{1}{R} \tilde{\Phi},_{R}+\tilde{\Phi}, Z Z=0 \\
\tilde{\Lambda}[\tilde{\Phi}]=\frac{1}{2} \int R\left[\left(\tilde{\Phi},_{R}^{2}-\tilde{\Phi},_{Z}^{2}\right) d R+2 \tilde{\Phi},_{R} \tilde{\Phi}_{, Z} d Z\right] .
\end{gathered}
$$

Note that (14) is the Laplace equation in flat three-dimensional space and so $\tilde{\Phi}$ can be taken as a solution of Laplace equation for an appropriated Newtonian source with axial symmetry. Once a solution $\tilde{\Phi}$ is known, $\tilde{\Lambda}$ is computed from (15) and so we obtain, from (12) - (13), a solution of the field equations (2) - (5).

By using (13) - (15) we find the nonzero components of the Riemann curvature tensor,

$$
\begin{aligned}
R_{r \varphi r \varphi} & =\frac{R^{2} R,,_{r}}{2 e^{\tilde{\Phi}}}\left[R,_{r}\left(\tilde{\Phi},_{Z}^{2}-\tilde{\Phi},_{R}^{2}\right)+2 R,,_{z} \tilde{\Phi},_{R} \tilde{\Phi},_{Z}\right], \\
R_{r \varphi z \varphi} & =\frac{R^{2} R,_{r}}{2 e^{\tilde{\Phi}}}\left[R,_{z}\left(\tilde{\Phi},_{Z}^{2}-\tilde{\Phi},_{R}^{2}\right)-2 R,_{r} \tilde{\Phi},_{R} \tilde{\Phi},_{Z}\right], \\
R_{z \varphi z \varphi} & =R_{r \varphi z \varphi},
\end{aligned}
$$

and the Kretschmann scalar

$$
\begin{aligned}
R_{c d}^{a b} R_{a b}^{c d} & =\frac{e^{2(\tilde{\Phi}-\tilde{\Lambda})}}{R,_{r}^{2}+R,_{z}^{2}} \times\left[R,_{z}^{2}\left(\tilde{\Phi},_{R}^{2}-\tilde{\Phi},_{Z}^{2}\right)^{2}\right. \\
& +R,_{r}^{2}\left(\tilde{\Phi},_{R}^{4}+6 \tilde{\Phi},_{R}^{2} \tilde{\Phi},_{Z}^{2}+\tilde{\Phi},_{Z}^{4}\right) \\
& \left.+4 R,_{r} R,_{z} \tilde{\Phi},_{R} \tilde{\Phi},_{Z}\left(\tilde{\Phi},_{R}^{2}-\tilde{\Phi},_{Z}^{2}\right)\right]
\end{aligned}
$$

From these expressions, using (9) and the properties of $\tilde{\Phi}$, we can test if the solutions have strong curvature singularities.

\subsection{The Energy-Momentum Tensor}

Due to the discontinuous behavior of the derivatives of the metric tensor across the disk, the Riemann curvature tensor contain Dirac delta functions. 
The energy-momentum tensor can be obtained by the distributional approach due to Papapetrou and Hamouni [19], Lichnerowicz [20], and Taub [21]. See also Israel [22, 23]. It can be written as $T^{a}{ }_{b}=\left[T^{a}{ }_{b}\right] \delta(z)$, where $\delta$ is the Dirac function with support on the disk and $\left[T^{a}{ }_{b}\right]$ is the distributional energymomentum tensor, which yield the volume energy density and the principal stresses. The "true" surface energy-momentum tensor of the disk can be written as $\tau_{b}^{a}=e^{(\Lambda-\Phi) / 2}\left[T^{a}{ }_{b}\right]$.

The discontinuities in the first derivatives of the metric tensor can be cast as [11, 24]

$$
b_{a b}=\left.g_{a b, z}\right|_{z=0^{+}}-\left.g_{a b, z}\right|_{z=0^{-}},
$$

then the distributional energy-momentum tensor is given by

$$
\left[T^{a}{ }_{b}\right]=\frac{1}{2}\left\{b^{a z} \delta^{z}{ }_{b}-b^{z z} \delta^{a}{ }_{b}+g^{a z} b^{z}{ }_{b}-g^{z z} b^{a}{ }_{b}+b^{c}{ }_{c}\left(g^{z z} \delta^{a}{ }_{b}-g^{a z} \delta^{z}{ }_{b}\right)\right\} .
$$

For the metric (1) we obtain $\left[T_{b}^{a}\right] \equiv \operatorname{diag}\left(-\epsilon, p_{\varphi}, p_{r}, 0\right)$, with

$$
\begin{aligned}
\epsilon & =e^{\Phi-\Lambda}\left[2 \Phi, z-\Lambda, z-\frac{2 R, z}{R}\right], \\
p_{\varphi} & =e^{\Phi-\Lambda}[\Lambda, z], \\
p_{r} & =e^{\Phi-\Lambda}\left[\frac{2 R, z}{R}\right],
\end{aligned}
$$

where all these quantities are evaluated at $z=0^{+}, 0 \leq r \leq 1$. In order to obtain expressions for a disk with non-unit radius, we only need to make the transformation $r \rightarrow a r$, where $a$ is the radius of the disk.

From (24) and (9) we can compute the general expression for the radial stress $p_{r}$,

$$
p_{r}= \pm \frac{2 \alpha \sqrt{1-r^{2}}}{1+\left(\alpha^{2}-1\right) r^{2}} e^{\tilde{\Phi}-\tilde{\Lambda}}
$$

where the \pm correspond to the two signs of the square root in (9). Thus, when we take the positive (negative) sign the stress is positive (negative), we have disks solutions with radial pressure (tension). From the fact that $\tilde{\Phi}$ and $\tilde{\Lambda}$ are both real and finite at the surface of the shells, we can see that $p_{r}$ is a well behaved function of $r$ for any value of $\alpha$. 
By doing $z=0,0 \leq r \leq 1$ in (10) and (11) we obtain the image of the disks by the conformal mapping,

$$
\alpha^{2} R^{2}+Z^{2}=\alpha^{2}
$$

so that the disks are mapped into spheroidal thin shells of matter. According with this, the solution (9) leads to three different boundary conditions for a three-dimensional potential problem. These boundary conditions correspond to the three possibilities for $\alpha$ : $\alpha=1$, a spherical shell, $\alpha>1$, a prolate spheroidal shell, and $0<\alpha<1$, an oblate spheroidal shell.

Using the above shells as sources to solve the Laplace equation (14) we obtain many different solutions of (2) - (5). In all the cases the solutions of (14) are known and can be written in terms of Legendre functions. Due to the discontinuous character of the Weyl coordinate $Z$ across the disk, a polynomial in odd powers of $Z$ is discontinuous across the disk while that a polynomial in even powers is a continuous function everywhere but with discontinuous first derivatives with respect to $z$ across the disk. Accordingly, the solutions of (14) must be chosen with the appropriated Legendre functions of even order in such away that the solutions of (2) - (5) have the assumed behavior at the disks.

In order to have a one-to-one correspondence in the outer region to the disks, we need to choose the appropriate sign of the square root in (9) that map this region completely into the exterior or interior region to the shells. The appropriate choose will depend of the value of $\alpha$. When $\alpha \neq 1$ we only can take the positive sign of the square root because with the negative sign the map (9) is not single valued at the exterior of the disk and, in this case, we only can take the solutions of (14) that correspond to the potential in the exterior of the appropriated spheroidal shell.

On the other hand, when $\alpha=1$ we can take separately both of the possibilities in (9) and, for each one, we will obtain a well posed boundary value problem. When we take the positive sign, the outer region to the disk is mapped into the outer region to a spherical shell of radius one. Thus we must take the solutions of (14) that correspond to the potential in the exterior of the shell. When we take the negative sign, the outer region to the disk is mapped into the interior of the same shell and so the solutions of (14) must be those that correspond to the interior potential. 


\section{Static Disks with Radial Pressure}

For the positive sign of the square root in (9) there are three different families of solutions corresponding to the three possibilities $\alpha=1, \alpha<1$ and $\alpha>$ 1. In each case we consider simple known Weyl solutions and from them, through (12) and (13), we obtain the corresponding thin disks solutions. As we shall see, in all the solutions considered we obtain disks with a well behaved central sector where the energy-momentum tensor agree with the weak and strong energy conditions [25], but with a border region where $\epsilon<0$ in violation of the weak energy condition.

\subsection{Chazy-Curzon Disks}

The first family of solutions is obtained by choosing $\alpha=1$ and transforming the disk into a spherical shell of radius one and the outer region of the disk into the outer region to the shell. To solve the Laplace equation (14) we introduce spherical coordinates $(\mathcal{R}, \theta)$, related to the Weyl coordinates by

$$
R=\mathcal{R} \sin \theta, \quad Z=\mathcal{R} \cos \theta
$$

with $0 \leq \mathcal{R} \leq \infty$ and $-\frac{\pi}{2} \leq \theta \leq \frac{\pi}{2}$. The shell is at $\mathcal{R}=1$.

The general solution for the Laplace equation (14) in the exterior of the shell, continuous across the disk, can be written as

$$
\tilde{\Phi}=-\sum_{n=0}^{\infty} \frac{2 C_{2 n} P_{2 n}(\cos \theta)}{\mathcal{R}^{2 n+1}}
$$

where $C_{2 n}$ are constants and $P_{2 n}(\cos \theta)$ are the usual Legendre polynomials. The simplest case is the Chazy-Curzon solution [26, 27]

$$
\tilde{\Phi}=-\frac{2 \gamma}{\mathcal{R}}, \quad \tilde{\Lambda}=-\frac{\gamma^{2} \sin ^{2} \theta}{\mathcal{R}^{2}},
$$

where $\gamma$ is a real constant. Note that $\tilde{\Phi}$ and $\tilde{\Lambda}$ are well behaved continuous functions for $\mathcal{R} \geq 1$ and vanish at infinity.

The metric tensor in Weyl coordinates is given by

$$
g_{t t}=-\exp \left\{\frac{-2 \gamma}{\sqrt{R^{2}+Z^{2}}}\right\},
$$




$$
\begin{aligned}
g_{\varphi \varphi} & =R^{2} \exp \left\{\frac{2 \gamma}{\sqrt{R^{2}+Z^{2}}}\right\}, \\
g_{r r} & =\left|W^{\prime}\right|^{2} \exp \left\{\frac{2 \gamma}{\sqrt{R^{2}+Z^{2}}}-\frac{\gamma^{2} R^{2}}{\left(R^{2}+Z^{2}\right)^{2}}\right\}, \\
g_{z z} & =g_{r r} .
\end{aligned}
$$

Using (10), (11) and (13), we can obtain its expression in the original coordinates. Then, taking $z=0^{+}$and using (22) - (24), (10) and (11), we obtain

$$
\begin{aligned}
\epsilon & =p_{r}\left[2(\gamma-1)-\gamma^{2} r^{2}\right], \\
p_{\varphi} & =p_{r}\left[1+\gamma^{2} r^{2}\right], \\
p_{r} & =p_{0} \sqrt{1-r^{2}} \exp \left\{\gamma^{2} r^{2}\right\},
\end{aligned}
$$

where $p_{0}$ is a positive constant and $0 \leq r \leq 1$.

In order to analyze the behavior of the above solution we consider the weak and strong energy conditions [25]. One of the requirements of the weak energy condition is that $\epsilon \geq 0$ everywhere. From (34) we see that this condition is equivalent to

$$
2(\gamma-1)-\gamma^{2} r^{2} \geq 0, \quad 0 \leq r \leq 1
$$

This equation has one zero at

$$
r_{0}^{2}=\frac{2(\gamma-1)}{\gamma^{2}}
$$

and so, in order to have $\epsilon>0$ for $r=0$, we must impose the condition $\gamma>1$. The maximum value for $r_{0}$ is obtained for $\gamma=2$ and is given by $r_{0}^{2}=1 / 2$.

The strong energy condition require that $\sigma \geq 0$, where $\sigma=\epsilon+p_{\varphi}+p_{r}$ is the effective Newtonian density. From (34) - (36) we see that $\sigma=2 \gamma p_{r}$ and so is positive everywhere on the disk for all value of $\gamma>0$. In Figure 1 we plot the functions $\sigma, \epsilon, p_{r}$ and $p_{\varphi}$ in the interval $0 \leq r \leq 1$ for this choice of the parameters, in $p_{0}$ units. The disk have a central region that satisfy the weak and strong energy conditions and a border region where $\epsilon<0$, in violation of the weak energy condition. 


\subsection{Zipoy-Voorhees Disks}

The second family of solutions is obtained by choosing $\alpha>1$ in (9), transforming the disk into a prolate spheroidal shell. To solve the Laplace equation (14) we introduce prolate spheroidal coordinates $(\xi, \eta)$, related to the Weyl coordinates by

$$
R^{2}=\kappa^{2}\left(\xi^{2}-1\right)\left(1-\eta^{2}\right), \quad Z=\kappa \xi \eta,
$$

where $\kappa=\sqrt{\alpha^{2}-1}, 1 \leq \xi \leq \infty$ and $0 \leq \eta \leq 1$. The shell is located at $\xi=\alpha / \sqrt{\alpha^{2}-1}>1$.

The general solution for the exterior of the shell, continuous across the disk, can be written as

$$
\tilde{\Phi}=-\sum_{n=0}^{\infty} 2 C_{2 n} P_{2 n}(\eta) Q_{2 n}(\xi),
$$

where $Q_{2 n}$ are the Legendre functions of second kind. The simplest case is the Zipoy-Voorhees solution [28, 29], also known as the Weyl $\gamma$-solution [5, 6], given by

$$
\tilde{\Phi}=\gamma \ln \frac{\xi-1}{\xi+1}, \quad \tilde{\Lambda}=\gamma^{2} \ln \frac{\xi^{2}-1}{\xi^{2}-\eta^{2}},
$$

where $\gamma$ is a real constant. We see that $\tilde{\Phi}$ and $\tilde{\Lambda}$ are continuous on the disk and vanish at infinity.

The metric tensor in the prolate spheroidal coordinates is given by

$$
\begin{aligned}
g_{t t} & =-\left[\frac{\xi-1}{\xi+1}\right]^{\gamma}, \\
g_{\varphi \varphi} & =R^{2}\left[\frac{\xi+1}{\xi-1}\right]^{\gamma}, \\
g_{r r} & =\left|W^{\prime}\right|^{2}\left[\frac{\xi^{2}-1}{\xi^{2}-\eta^{2}}\right]^{\gamma^{2}}\left[\frac{\xi+1}{\xi-1}\right]^{\gamma}, \\
g_{z z} & =g_{r r} .
\end{aligned}
$$

Using (10), (11), (39) and (13), we can obtain expressions in the original coordinates. Taking the values of the above expressions at the disk and 
using (22) - (24) we compute the energy density and pressures,

$$
\begin{aligned}
\epsilon & =p_{r}\left[\frac{2 \gamma \sqrt{\alpha^{2}-1}-\alpha}{\alpha}-\frac{1+\left(\alpha^{2}-1\right) \gamma^{2} r^{2}}{1+\left(\alpha^{2}-1\right) r^{2}}\right] \\
p_{\varphi} & =p_{r}\left[\frac{1+\left(\alpha^{2}-1\right) \gamma^{2} r^{2}}{1+\left(\alpha^{2}-1\right) r^{2}}\right] \\
p_{r} & =p_{0} \sqrt{1-r^{2}}\left[1+\left(\alpha^{2}-1\right) r^{2}\right]^{\left(\gamma^{2}-1\right)}
\end{aligned}
$$

where $p_{0}$ is a positive constant and $0 \leq r \leq 1$.

From (46) we obtain the conditions

$$
\beta=\frac{\gamma \sqrt{\alpha^{2}-1}}{\alpha} \geq 1
$$

to have $\epsilon \geq 0$ at $r=0$, and

$$
\alpha^{2} \beta^{2}-2 \alpha^{2} \beta+\left(\alpha^{2}+1\right) \leq 0
$$

in order to have $\epsilon \geq 0$ at $r=1$. We see that $\gamma$ and $\alpha$ can not be adjusted to have $\epsilon \geq 0$ at the border of the disk and so $\epsilon(r)$ have a zero at $r_{0}$ given by

$$
r_{0}^{2}=\frac{2 \alpha^{2}(\beta-1)}{\beta^{2}-\left(1-\alpha^{2}\right)(2 \beta-1)} .
$$

Now, to have $\epsilon\left(r_{0}\right)=0$ with real values of $\gamma$ and $\alpha$, we obtain the conditions

$$
\alpha \leq \frac{1-r_{0}^{2}}{r_{0}^{2}}, \quad 0<r_{0}^{2}<\frac{1}{2} .
$$

The value of $\beta$ is given by

$$
\beta=\frac{1}{\alpha^{2} r_{0}^{2}}\left[1+\left(\alpha^{2}-1\right) r_{0}^{2} \pm \sqrt{\left(1-r_{0}^{2}\right)^{2}-\alpha^{2} r_{0}^{4}}\right]
$$

Then, by choosing a value of $r_{0}<1 / \sqrt{2}$ we obtain values for $\alpha, \beta$ and $\gamma$ so that the solution satisfy the weak and strong energy conditions in the central region of the disk, for $r<r_{0}$. 
By choosing $r_{0}^{2}=0.2$ we can take $\alpha=4, \beta=5 / 4$ and $\gamma=\sqrt{5 / 3}$ so that the radial pressure is given by

$$
p_{r}=p_{0} \sqrt{1-r^{2}}\left(1+15 r^{2}\right)^{\frac{2}{3}} .
$$

From (46) - 48) we can see that $\sigma=2 \beta p_{r}>0$ so that the solution satisfy the weak and strong energy conditions in the central region of the disk. In Figure 2 we plot the functions $\epsilon, p_{r}, p_{\varphi}$ and $\sigma$ in the interval $0 \leq r \leq 1$ for this choice of the parameters, in $p_{0}$ units. As in the first solution, the disk have a central region that satisfy the weak and strong energy conditions and a border region where $\epsilon<0$, in violation of the weak energy condition.

\subsection{Bonnor and Sackfield Disks}

Finally, the third family of solutions is obtained by choosing $0<\alpha<1$ in (9) and transforming the disk into a oblate spheroidal shell. To solve the Laplace equation (14) we introduce oblate spheroidal coordinates $(\zeta, \eta)$, related to the Weyl coordinates by

$$
R^{2}=\kappa^{2}\left(\zeta^{2}+1\right)\left(1-\eta^{2}\right), \quad Z=\kappa \zeta \eta
$$

where $\kappa=\sqrt{1-\alpha^{2}}, 0 \leq \zeta \leq \infty$ and $0 \leq \eta \leq 1$. The shell is at $\zeta=$ $\alpha / \sqrt{1-\alpha^{2}}>0$.

The general solution for the exterior of the shell, continuous across the disk, can be written as

$$
\tilde{\Phi}=-\sum_{n=0}^{\infty} 2 C_{2 n} P_{2 n}(\eta) q_{2 n}(\zeta)
$$

where $q_{2 n}(\zeta)=i^{2 n+1} Q_{2 n}(i \zeta)$. The simplest case is given by

$$
\tilde{\Phi}=-2 \gamma \cot ^{-1} \zeta, \quad \tilde{\Lambda}=-\gamma^{2} \ln \frac{\zeta^{2}+1}{\zeta^{2}+\eta^{2}}
$$

where $\gamma$ is a real constant. As in the above two solutions, $\tilde{\Phi}$ and $\tilde{\Lambda}$ are continuous on the disk and vanish at infinity. The above solution was found by Zipoy [28] and Voorhees [29] and interpreted by Bonnor and Sackfield [1] as the gravitational field of a pressureless disk. We shall see that the method 
based in the conformal transformation not only leads to a nonzero radial pressure, but also to a nonzero azimuthal pressure.

From the metric tensor in oblate spheroidal coordinates

$$
\begin{aligned}
g_{t t} & =-\exp \left\{-2 \gamma \cot ^{-1} \zeta\right\}, \\
g_{\varphi \varphi} & =R^{2} \exp \left\{2 \gamma \cot ^{-1} \zeta\right\}, \\
g_{r r} & =\left|W^{\prime}\right|^{2}\left[\frac{\zeta^{2}+\eta^{2}}{1+\zeta^{2}}\right]^{\gamma^{2}} \exp \left\{2 \gamma \cot ^{-1} \zeta\right\}, \\
g_{z z} & =g_{r r},
\end{aligned}
$$

we can obtain, using (10), (11]), (55) and (13), its expression in the original coordinates. Taking the values of the above expressions at the disk and using (22) - (24) we compute the energy density and pressures

$$
\begin{aligned}
\epsilon & =p_{r}\left[\frac{2 \gamma \sqrt{1-\alpha^{2}}-\alpha}{\alpha}-\frac{1+\left(1-\alpha^{2}\right) \gamma^{2} r^{2}}{1+\left(\alpha^{2}-1\right) r^{2}}\right] \\
p_{\varphi} & =p_{r}\left[\frac{1+\left(1-\alpha^{2}\right) \gamma^{2} r^{2}}{1+\left(\alpha^{2}-1\right) r^{2}}\right] \\
p_{r} & =\frac{p_{0} \sqrt{1-r^{2}}}{\left[1+\left(\alpha^{2}-1\right) r^{2}\right]\left(\alpha^{2}+1\right)}
\end{aligned}
$$

where $p_{0}$ is a positive constant and $0 \leq r \leq 1$.

From (62) we obtain the condition

$$
\beta=\frac{\gamma \sqrt{1-\alpha^{2}}}{\alpha} \geq 1
$$

to have $\epsilon \geq 0$ at $r=0$. As in the second family of solutions, the condition (50) is imposed in order to have $\epsilon \geq 0$ at $r=1$. Again, $\gamma$ and $\alpha$ can not be adjusted to have $\epsilon \geq 0$ at the border of the disk and so $\epsilon(r)$ have a zero at $r_{0}$ given by (51). Now, to have $\epsilon\left(r_{0}\right)=0$ with real values of $\gamma$ and $\alpha$, we obtain

$$
\alpha \leq \frac{1-r_{0}^{2}}{r_{0}^{2}}, \quad \frac{1}{2}<r_{0}^{2}<1
$$


so that the value of $\beta$ is again given by (53). Then, by choosing a value of $1 / \sqrt{2}<r_{0}<1$ we obtain limits for $\gamma$ and $\alpha$ so that the solution satisfy the weak and strong energy conditions in the central region of the disk, $r<r_{0}$.

By choosing $r_{0}^{2}=0.8$ we can take $\alpha=1 / 4, \beta=5$ and $\gamma=\sqrt{5 / 3}$ so that the radial pressure is given by

$$
p_{r}=\frac{p_{0} \sqrt{1-r^{2}}}{\left(1-\frac{15}{16} r^{2}\right)^{\frac{8}{3}}} .
$$

From (46) - (48) we can see that $\sigma=2 \beta p_{r}>0$ and so again we have a solution that satisfy the weak and strong energy conditions for $0 \leq r \leq r_{0}$. In Figure 3 we plot the functions $\epsilon, p_{r}, p_{\varphi}$ and $\sigma$ in the interval $0.85 \leq r \leq 1$ for this choice of the parameters, in $p_{0}$ units. As in the above two solutions, the disk have a central region that satisfy the weak and strong energy conditions and a border region where $\epsilon<0$, in violation of the weak energy condition. However, in this case the central well behaved region can be extended to practically the entire disk by an appropriate selection of the parameters $\gamma$ and $\alpha$.

\section{Statics disks with Radial Tension}

We now find some solutions with radial tension by taking the negative sign of the square root in (9). There is only one family of solutions, corresponding to the case $\alpha=1$, obtained by transforming the disk into a spherical shell of radius one and the outer region of the disk into the inner region to the shell.

To solve the Laplace equation (14) we again introduce the spherical coordinates (27). The general solution for the Laplace equation (14) in the interior of the shell, continuous across the disk, can be written as

$$
\tilde{\Phi}=-\sum_{n=0}^{\infty} 2 C_{2 n} \mathcal{R}^{2 n} P_{2 n}(\cos \theta)
$$

where $C_{2 n}$ are constants. The simplest case is obtained by taking the first term of the series and is given by

$$
\tilde{\Phi}=-2 \gamma, \quad \tilde{\Lambda}=0
$$

where $\gamma$ is a real constant. 
The metric tensor is given by

$$
\begin{aligned}
& g_{t t}=-e^{-2 \gamma}, \\
& g_{\varphi \varphi}=R^{2} e^{2 \gamma}, \\
& g_{r r}=\left|W^{\prime}\right|^{2} e^{2 \gamma}, \\
& g_{r r}=g_{z z},
\end{aligned}
$$

where $R(r, z)$ and $\left|W^{\prime}(\omega)\right|$ are obtained from (10) and (11).

From (16) and (17), we see that $R^{a b}{ }_{c d} \equiv 0$ and so we have a spacetime defect [24]. The energy density and tensions are given by

$$
\epsilon=-2 p_{\varphi}=-2 p_{r}=2 p_{0} \sqrt{1-r^{2}}
$$

where $p_{0}$ is a positive constant and $0 \leq r \leq 1$. In this case we have $\sigma=0$ and we can see that this solution satisfy all the energy conditions everywhere on the disk. This disk can be interpreted as being formed by cosmic strings located along the radial direction interwoven with loops. Another disklike topological defect, an infinite disk with zero radial stress, was presented in [12]. The energy density and the azimuthal pressure were given by $\epsilon=$ $p_{\varphi}=1$, also in agreement with the energy conditions.

A second solution is obtained by taking the second term of the series in (68). We find

$$
\tilde{\Phi}=\gamma\left(R^{2}-2 Z^{2}\right), \quad \tilde{\Lambda}=\frac{\gamma^{2} R^{2}}{2}\left(R^{2}-8 Z^{2}\right)
$$

where $\gamma$ is a real constant. As in the other solutions, $\tilde{\Phi}$ and $\tilde{\Lambda}$ are continuous across the disk and vanish at infinity, where $R=Z=0$.

The metric tensor in Weyl coordinates is given by

$$
\begin{aligned}
g_{t t} & =-\exp \left\{\gamma R^{2}-2 \gamma Z^{2}\right\}, \\
g_{\varphi \varphi} & =R^{2} \exp \left\{2 \gamma Z^{2}-\gamma R^{2}\right\},
\end{aligned}
$$




$$
\begin{aligned}
g_{r r} & =\left|W^{\prime}\right|^{2} \exp \left\{\frac{\gamma^{2} R^{4}}{2}-4 \gamma^{2} R^{2} Z^{2}-\gamma R^{2}+2 \gamma Z^{2}\right\} \\
g_{z z} & =g_{r r}
\end{aligned}
$$

Expressions in the original coordinates are obtained using (10), (11), and (13).

Taking the values of the above expressions at the disk and using (22) (24) we compute the energy density and pressures,

$$
\begin{aligned}
\epsilon & =-p_{r}\left[9 \gamma^{2} r^{4}-(6+8 \gamma) \gamma r^{2}+2(2 \gamma+1)\right], \\
p_{\varphi} & =-p_{r}\left[8 \gamma^{2} r^{2}-9 \gamma^{2} r^{4}-1\right] \\
p_{r} & =-p_{0} \exp \left\{(3+4 \gamma) \gamma r^{2}-4.5 \gamma^{2} r^{4}\right\} \sqrt{1-r^{2}}
\end{aligned}
$$

where $p_{0}$ is a positive constant and $0 \leq r \leq 1$.

From (80) we can see that the weak energy condition is equivalent to

$$
9 \gamma^{2} r^{4}-(6+8 \gamma) \gamma r^{2}+2(2 \gamma+1) \geq 0
$$

for $0 \leq r \leq 1$. This expression have two zeros at

$$
r_{0}^{2}=\frac{3+4 \gamma \pm \sqrt{16 \gamma^{2}-12 \gamma-9}}{9 \gamma},
$$

and so there are three different classes of solutions corresponding to the values of $\gamma$ such that the above equation have either two, one or any real positive root.

When $\gamma<-0.5$ there is a solution $r_{0}<1$ of (84) in such away that the disks have central regions with $\epsilon<0$ and border regions with $\epsilon>0$. When $-0.5 \leq \gamma \leq 3(1+\sqrt{5}) / 8$ we can see that $\epsilon \geq 0$ everywhere on the disks, accordingly the weak energy condition is satisfied. Finally, when $\gamma>$ $3(1+\sqrt{5}) / 8$, there are two different real positive solutions of (84), $r_{1}$ and $r_{2}$, so that the disks have a central region with $\epsilon>0$, when $r<r_{1}$, a region with $\epsilon<0$, when $r_{1}<r<r_{2}$, and a region with $\epsilon>0$, when $r>r_{2}$.

From $(80)-(82)$ we can see that

$$
\sigma=-\gamma p_{r}\left(4-6 r^{2}\right)
$$


Hence $\sigma=0$ at $r^{2}=2 / 3$. Accordingly, the solutions are in agree with the strong energy condition only in a region, the central region of the disks when $\gamma>0$, or the border region of the disks when $\gamma<0$. We also see from (81) that the azimuthal stress is zero at

$$
r_{ \pm}^{2}=\frac{4 \gamma \pm \sqrt{16 \gamma^{2}-9}}{9 \gamma} .
$$

When $|\gamma|>0.75$ there are two different positive roots, $r_{-}<r_{+}<1$. Hence there is a region of the disks where the azimuthal stress is positive, for $r_{-}<$ $r<r_{+}$. On the other hand, when $|\gamma|<0.75$ the azimuthal stress is negative everywhere.

In order to show the behavior of the different classes of solutions that can be obtained we consider some particular values of $\gamma$. In Figure $4-7$ we plot the functions $\epsilon, p_{r}, p_{\varphi}$ and $\sigma$ in the interval $0 \leq r \leq 1$ for $\gamma=-1, \gamma=-0.5$, $\gamma=1$ and $\gamma=2$, respectively, in $p_{0}$ units. We see that very different classes of solutions can be obtained, some of them with either the center of the disk or the border in accord with the energy conditions.

\section{Concluding Remarks}

In this work a method was presented which can be used to obtain static thin disks models with a well behaved nonzero radial stress. The general expression that was obtained for the radial stress admits solutions with positive stress, representing disks with radial pressure support, and solutions with negative stress, representing disks with radial tension support. Is easy to shown that, with the simple solution (9), when we consider solutions with positive stress is not possible to fulfill all the energy conditions at the entire disk, whereas that with negative stress we can obtain solutions that satisfy all the energy conditions everywhere on the disks, as the first solution studied at Sec. 4.

The method that was described can be used to obtain many static disks solutions with a radial pressure support that presents a very reasonable physical behavior and perhaps, by considering another more involved solutions of the Laplace equation (2), the solution can be adjusted to fulfill the weak and strong energy conditions everywhere on the disks, leading so to a physically acceptable source which would produce the considered vacuum solutions. 
Also, as we will do in a future paper, the method can be extended to consider rotating thin disks, which have a stronger astrophysical interest.

\section{Acknowledgments}

G.A.G. thanks a grant of CAPES - Brazil and a grant of COLCIENCIAS Colombia. P.S.L. thanks grants of CNPq and FAPESP - Brazil.

\section{References}

[1] W. A. Bonnor and A. Sackfield, Comm. Math. Phys. 8, 338 (1968)

[2] T. Morgan and L. Morgan, Phys. Rev. 183, 1097 (1969)

[3] L. Morgan and T. Morgan, Phys. Rev. D2, 2756 (1970)

[4] A. Chamorro, R. Gregory and J. M. Stewart, Proc. R. Soc. Lond. A413, $251(1987)$

[5] H. Weyl, Ann. Phys. 54, 117 (1917)

[6] H. Weyl, Ann. Phys. 59, 185 (1919)

[7] P. S. Letelier and S. R. Oliveira, J. Math. Phys. 28, 165 (1987)

[8] D. Lynden-Bell and S. Pineault, Mon. Not. R. Astron. Soc. 185, 679 (1978)

[9] J. P. S. Lemos, Class. Quan. Grav. 6, 1219 (1989)

[10] J. P. S. Lemos and P. S. Letelier, Class. Quan. Grav. 10, L75 (1993)

[11] J. P. S. Lemos and P. S. Letelier, Phys. Rev D49, 5135 (1994)

[12] J. P. S. Lemos and P. S. Letelier, Int. J. Mod. Phys. D5, 53 (1996)

[13] C. Klein, Class. Quan. Grav. 14, 2267 (1997)

[14] J. Bičák, D. Lynden-Bell and J. Katz, Phys. Rev. D47, 4334 (1993) 
[15] J. Bičák, D. Lynden-Bell and C. Pichon, Mon. Not. R. Astron. Soc. 265, 126 (1993)

[16] C. Pichon and D. Lynden-Bell, Mon. Not. R. Astron. Soc. 280, 1007 (1996)

[17] R. M. Wald. General Relativity. The University of Chicago Press, Chicago (1980)

[18] D. Kramer, H. Stephani, M. McCallum and E. Herlt. Exact Solutions of Einstein's Field Equations. Cambridge University Press, Cambridge (1980)

[19] A. Papapetrou and A. Hamouni, Ann. Inst. Henri Poincaré 9, 179 (1968)

[20] A. Lichnerowicz, C.R. Acad. Sci. 273, 528 (1971)

[21] A. H. Taub, J. Math. Phys. 21, 1423 (1980)

[22] W. Israel, Nuovo Cimento B 44, 1 (1966)

[23] W. Israel, Nuovo Cimento B 48, 463 (1967)

[24] P. S. Letelier and A. Wang, J. Math. Phys. 36, 3023 (1995)

[25] S. W. Hawking and G. F. R. Ellis. The Large Scale Structure of SpaceTime. Cambridge University Press, Cambridge (1973)

[26] J. Chazy, Bull. Soc. Math. France 52, 17 (1924)

[27] H. E. J. Curzon, Proc. London Math. Soc. 23, 477 (1924)

[28] D. M. Zipoy, J. Math. Phys. 7, 1137 (1966)

[29] B. H. Voorhees, Phys. Rev. D2, 2119 (1970) 


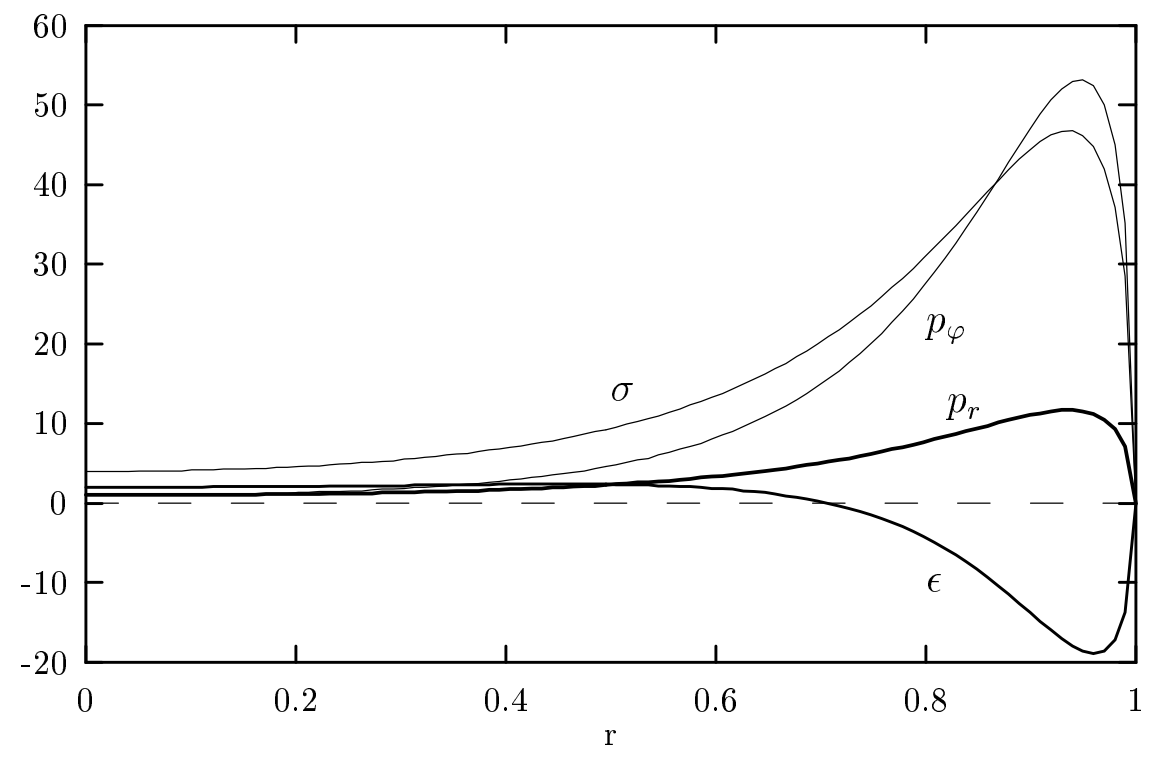

Figure 1: $\sigma, \epsilon, p_{r}$ and $p_{\varphi}$ for $\alpha=1$ and $\gamma=2$ in $p_{0}$ units of a disk of radius one. 


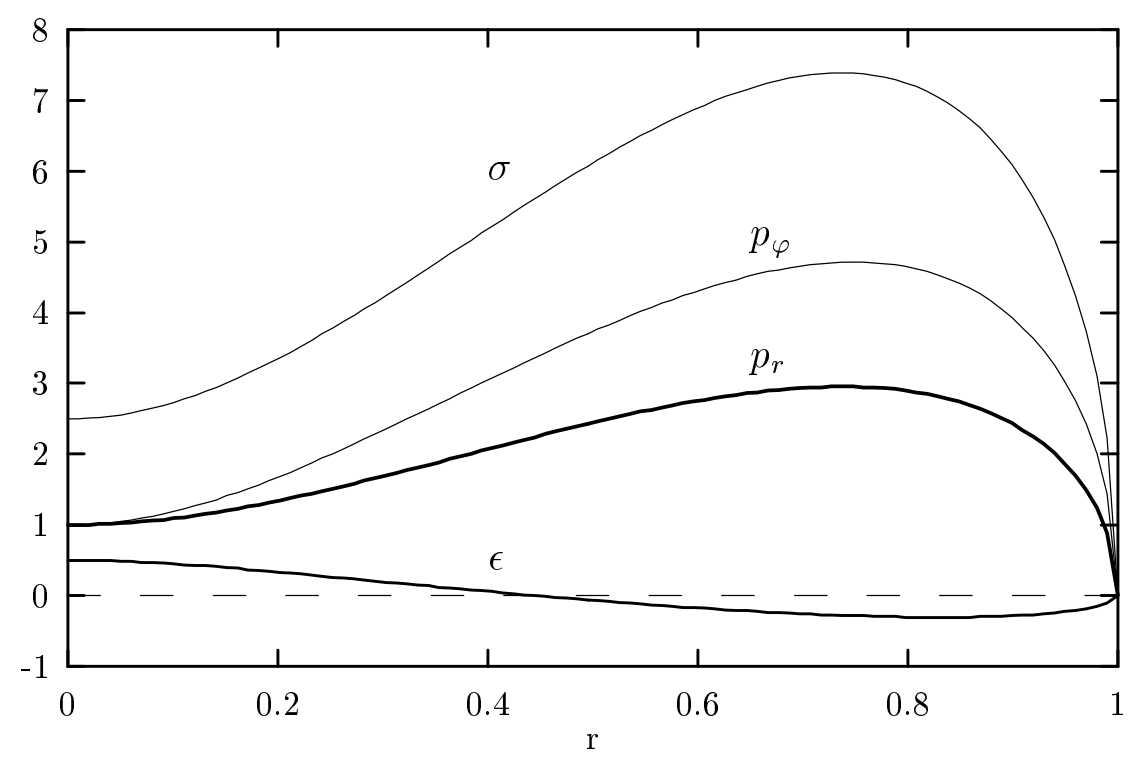

Figure 2: $\sigma, \epsilon, p_{r}$ and $p_{\varphi}$ for $\alpha=4, \beta=5 / 4$ and $\gamma=\sqrt{5 / 3}$ in $p_{0}$ units of a disk of radius one. 


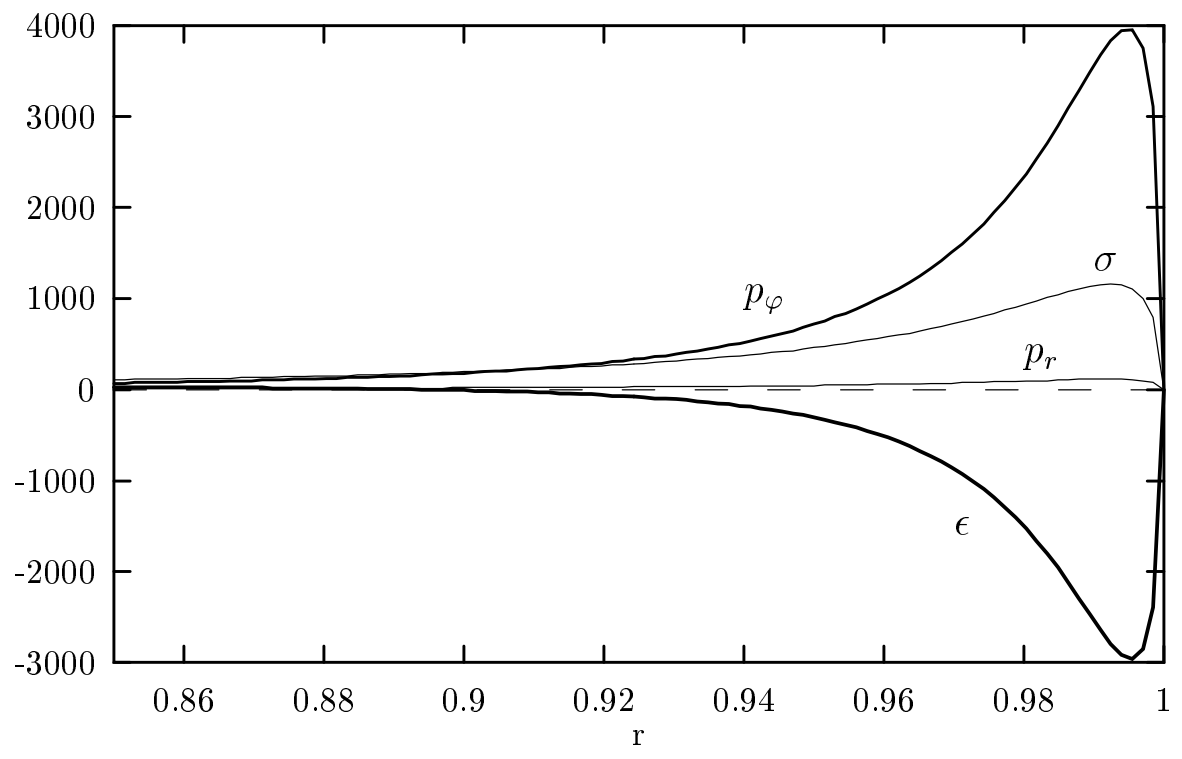

Figure 3: $\sigma, \epsilon, p_{r}$ and $p_{\varphi}$ for $\alpha=1 / 4, \beta=5$ and $\gamma=\sqrt{5 / 3}$ in $p_{0}$ units of a disk of radius one. 


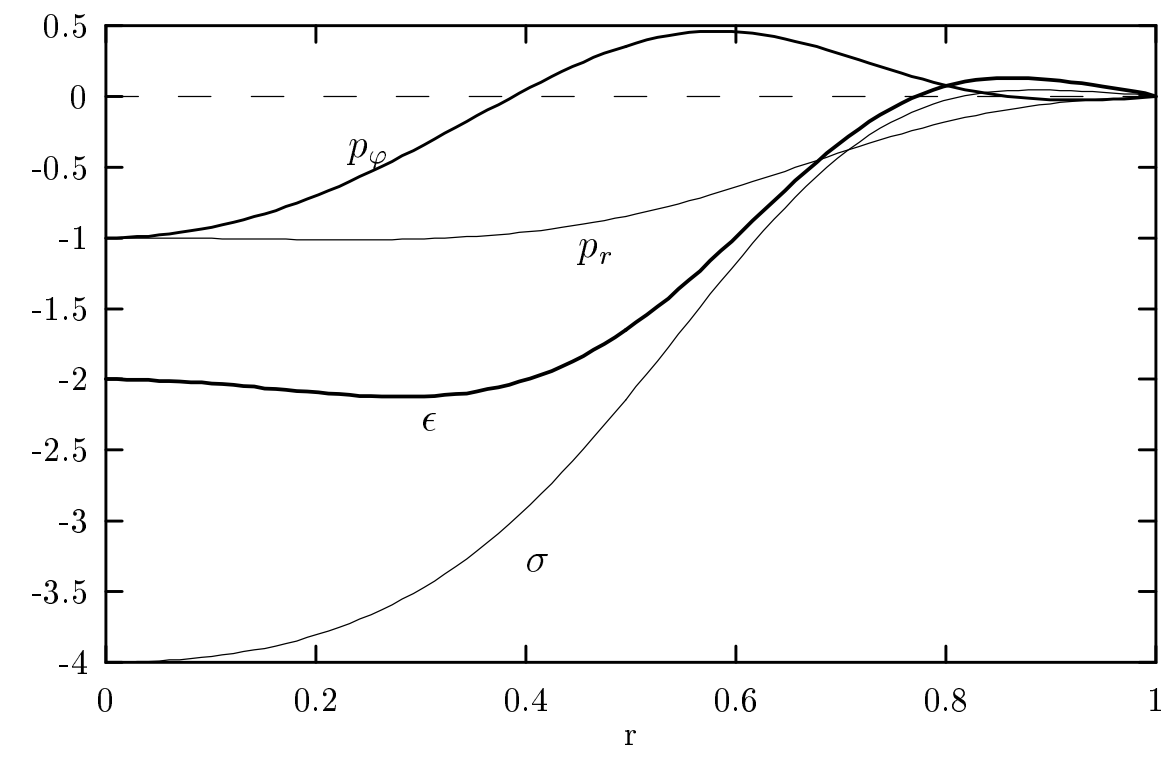

Figure 4: $\sigma, \epsilon, p_{r}$ and $p_{\varphi}$ for $\alpha=1$ and $\gamma=-1$ in $p_{0}$ units of a disk of radius one. 


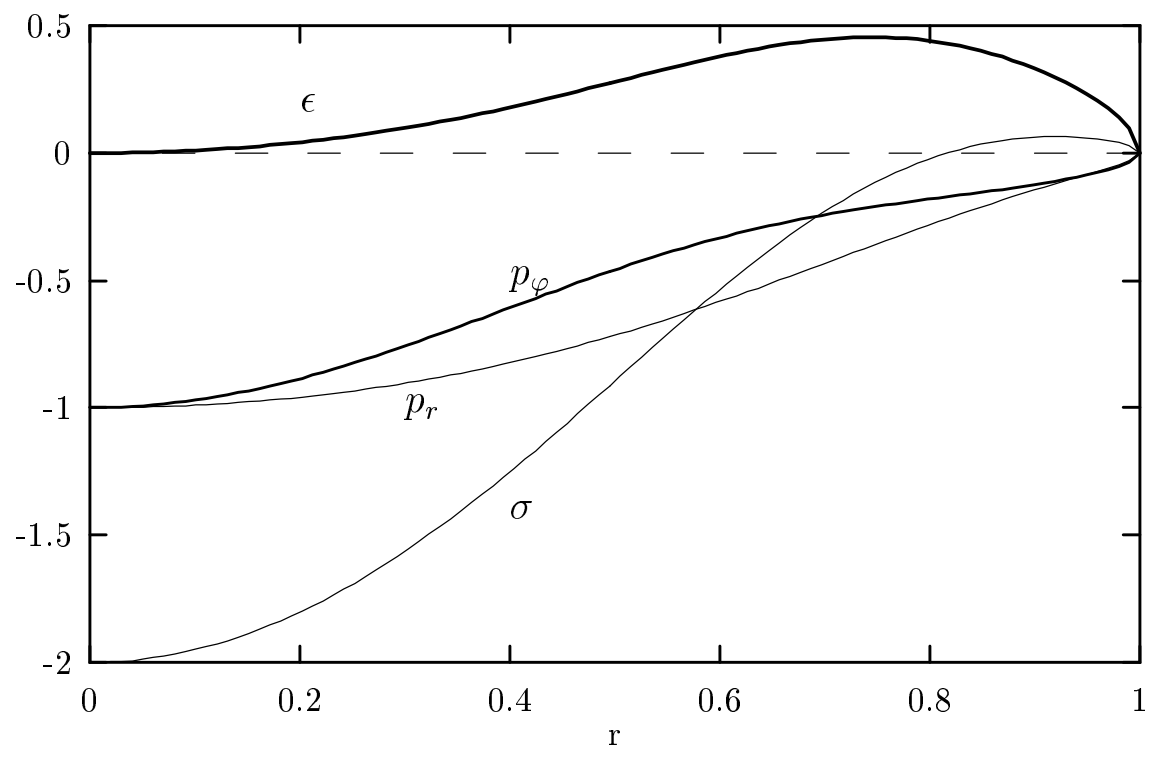

Figure 5: $\sigma, \epsilon, p_{r}$ and $p_{\varphi}$ for $\alpha=1$ and $\gamma=-0.5$ in $p_{0}$ units of a disk of radius one. 


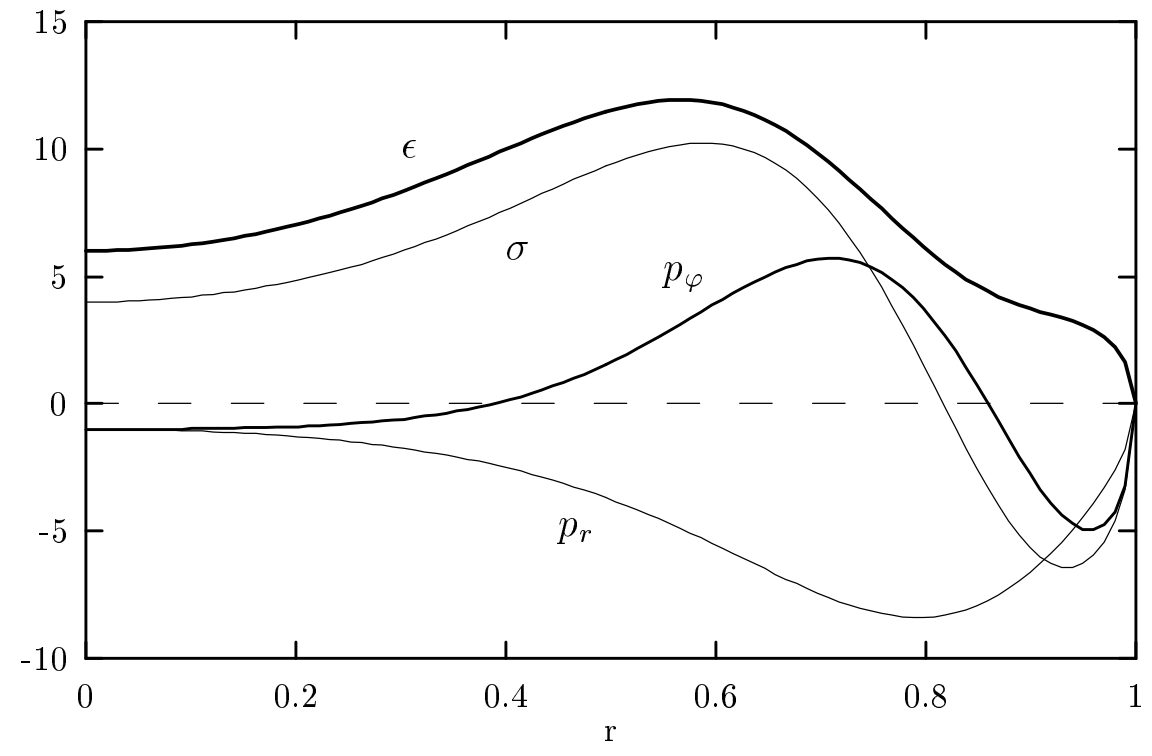

Figure 6: $\sigma, \epsilon, p_{r}$ and $p_{\varphi}$ for $\alpha=1$ and $\gamma=1$ in $p_{0}$ units of a disk of radius one. 


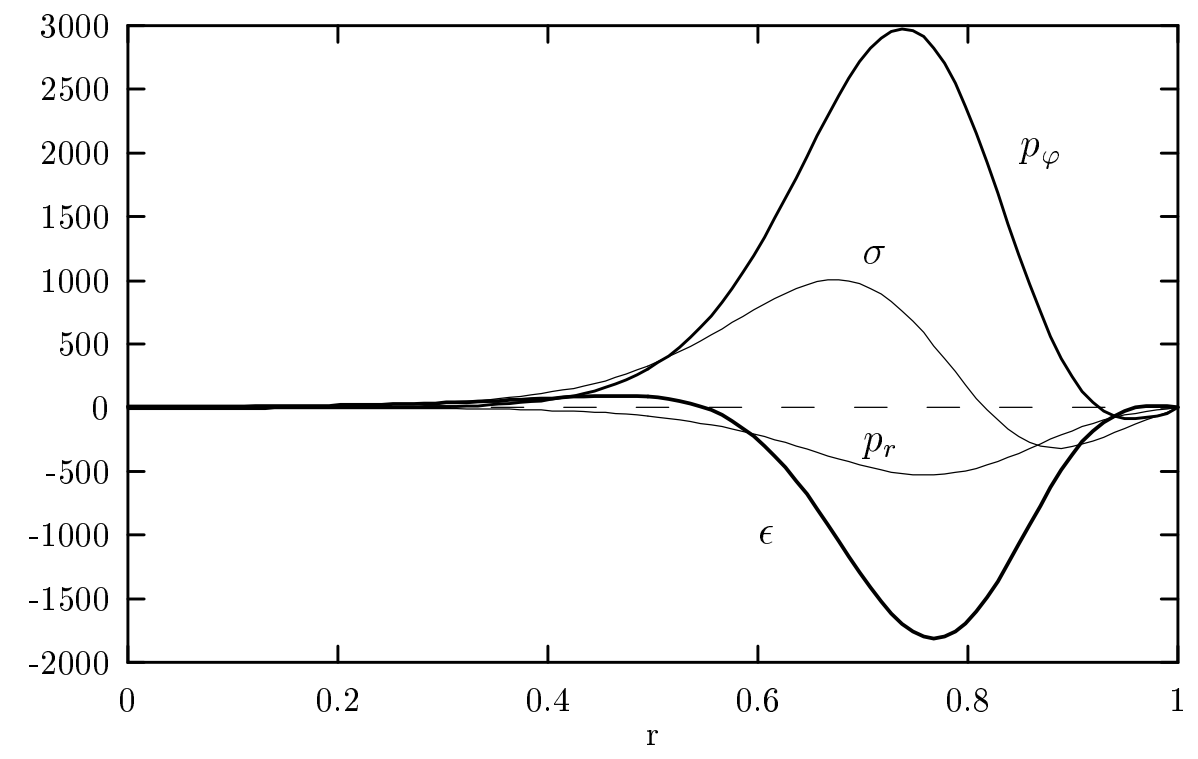

Figure 7: $\sigma, \epsilon, p_{r}$ and $p_{\varphi}$ for $a=1$ and $\gamma=2$ in $p_{0}$ units of a disk of radius one. 